\title{
Hacia la elaboración de un modelo de acompañamiento comunitario intercultural postdesarrollista
}

\author{
Towards the elaboration of a Post - Developmental Intercultural \\ Community Accompaniment Model
}

Sandra Carolina Rojas Hooker ${ }^{1}$

\section{Resumen}

El presente documento ha consistido en identificar elementos fundamentales para perfilar un modelo de acompañamiento comunitario intercultural postdesarrollista a saber: sentido de comunidıad, liderazgo comunitario, apropiación comunitaria, conciencia ambiental, establecimiento de redes y sostenibilidad. La investigación fue cualitativa desde un enfoque hermenéutico.

Por tanto, ha incorporado acciones que conllevan al buen vivir de los pueblos donde las articulaciones internas y externas son de vital importancia, siguiendo los principios de participación democrática, diálogo horizontal, construcción colectiva y corresponsabilidad que la comunidad demanda con miras a aportar, debatir y sobre todo decidir el futuro de la comunidad de manera conjunta. En este proceso, la conciencia ambiental juega un papel preponderante ya que se debe establecer una nueva relación de equilibrio entre los seres humanos y la naturaleza con miras a lograr la sostenibilidad ambiental, la justicia social y el bienestar tanto individual como colectivo.

Palabras clave: acompañamiento comunitario; buen vivir; sentido de comunidad; interculturalidad; conciencia ambiental; modelo.

\section{Summary}

This document has consisted of identifying fundamental elements to outline a postdevelopmental intercultural community accompaniment model, namely: sense of community, community leadership, community ownership, environmental awareness, networking and sustainability. The research was qualitative from a hermeneutical approach.

Therefore, it has incorporated actions that lead to the good living of peoples where internal and external articulations are of vital importance, following the principles

1 Máster en Estudios Indigenas, Directora del Instituto de Estudios y Promoción de la Autonomía de la Universidad de las Regiones Autónomas de la Costa Caribe Nicaragüense. Correo electrónico: sandra.rojas@uraccan.edu.ni, No. ID Orchid oooo-0002-7467-2366

Recibido: 02/04/2020 Aprobado: 28/05/2020

Rojas Hooker, S. (2020). Hacia la elaboración de un modelo de acompañamiento comunitario intercultural postdesarrollista. Ciencia $E$ 


\section{CIENCIAS SOCIALES}

of democratic participation, horizontal dialogue, collective construction and coresponsibility that the community demands with a view to contributing, debating and above all deciding the future of the community together. In this process, environmental awareness plays a preponderant role since a new balance relationship must be established between human beings and nature with a view to achieving environmental sustainability, social justice and well-being, both individually and collectively.

Keywords: Community accompaniment; Good Living; Community Significance; Interculturality; environmental awareness; Model.

\section{Introducción}

El presente artículo responde al trabajo final de módulo "Paradigma y Teoría del Desarrollo y Post Desarrollo" del Programa de Doctorado en Estudios Interculturales desarrollado en URACCAN en junio del 2019. Mediante el análisis documental se analizó, significó y resignificó de acuerdo a contextos particulares los conceptos claves de acompañamiento comunitario, enfoque de derechos humanos, interculturalidad y buen vivir con miras a perfilar una propuesta de modelo de acompañamiento comunitario intercultural posdesarrollista. La investigación es cualitativa desde un enfoque hermenéutico.

Al hablar de un modelo de acompañamiento comunitario intercultural desde elementos postdesarrollistas tomamos en cuenta una serie de mecanismos y estrategias que se utilizan para el logro de metas que marcan el caminar de los pueblos y comunidades en la búsqueda de su bienestar integral y sostenible.

Para realizar procesos de acompañamiento es necesario establecer objetivos y prioridades dentro de un plan de trabajo, diseñado con el protagonismo de las comunidades que responda a un propósito de cambio y de mejora para la colectividad para la satisfacción de necesidades actuales sin comprometer la capacidad de generaciones futuras de satisfacer las suyas.

El presente escrito tiene como finalidad identificar aspectos fundamentales en el proceso de acompañamiento que se realiza desde la URACCAN con miras a fortalecer las capacidades y potencialidades de los comunitarios para lograr el buen vivir como objetivo de vida deseable de los Pueblos y Comunidades en armonía con la madre naturaleza y en consonancia con los principios de igualdad y no discriminación.

\section{Revisión de literatura}

Se realizó una revisión literaria teniendo en cuenta varios factores entre las que se destacan el concepto de acompañamiento comunitario donde el Dr. Saballos (2016) 
y la Universidad Comunitaria Intercultural URACCAN (2017), nos brindan conceptos y elementos fundamentales para el desarrollo de la misma.

El siguiente factor se relaciona con el concepto de buen vivir como filosofía de vida para logar el bienestar en el ámbito familiar y colectivo donde los valores sociales juegan un papel preponderante (Carpio, 2014).

Finalmente, la interculturalidad y sus retos epistemológicos, donde autores como Valdividia Pinto (2006) y Fornet-Betancour (1994), hacen hincapié en el reconocimiento no sólo de las diferencias, sino en los elementos en común que tienen las diferentes culturas para construir una agenda en común donde el dialogo intercultural debe basarse en una relación igualitaria.

Para ello debe de tomarse en cuenta el enfoque basado en los Derechos Humanos como marco conceptual con miras al empoderamiento de los pueblos y comunidades para el fortalecimiento de su ciudadanía intercultural.

\section{Material y método}

Este artículo está enmarcado en la investigación cualitativa desde la investigación documental, que se caracteriza por la revisión de documentos que tengan los conceptos sobre el tema. En este sentido, se trabajo con información cualitativa y es de naturaleza hermenéutica, al interpretar y sintetizar los aportes de los autores para generar un nuevo saber.

\section{Acercamiento conceptual al modelo de acompañamiento comunitario al buen vivir desde elementos post- desarrollistas}

\section{a. Acompañamiento comunitario}

El acompañamiento connota una voluntad compartida de los que marchan juntos, confianza para hacer confluir estas voluntades, determinada claridad hacia dónde se va, un compromiso de caminar lo necesario (perspectiva de largo plazo), horizontalidad en el relacionamiento y sinergia al complementarse fuerzas que hace más efectivo el llegar al destino (Saballos 2016: 104).

Para URACCAN (2017:15) el acompañamiento comunitario intercultural es un proceso colaborativo, participativo, concertado e inclusivo, sustentado en el diálogo de saberes y haceres, mediante la articulación entre Universidad-Comunidad-Sociedad, que busca transformaciones estructurales, sociales, económicas, políticas, culturales y ambientales de los sistemas que sustentan las desigualdades en torno a la autonomía y autodeterminación de los pueblos, en pro del desarrollo con identidad y el ejercicio efectivo de las ciudadanías interculturales de género, para la construcción colectiva 


\section{CIENCIAS SOCIALES}

del buen vivir de los pueblos, el respeto, equilibrio y armonía con la Madre Tierra en el ámbito nacional e internacional.

\section{b. Buen vivir}

El buen vivir puede entenderse como vida en plenitud. Es una filosofía de vida basada en el paradigma comunitario, un sistema complejo y articulado de elementos filosóficos, políticos, culturales y éticos que atraviesan las relaciones primordiales que constituyen la comunidad y le dan sentido estructural como entidad ecosocial. Es una totalidad holística pensada y concreta que no puede ser entendida a través de elementos aislados sino en su integralidad. La bisagra entre lo espiritual y material es el trabajo a nivel individual, familiar y comunitario, pues solo este puede garantizar el buen vivir, el bienestar. En todos los niveles las formas de cooperación y solidaridad afianzan las relaciones primordiales en la vida comunitaria donde prevalece el sentido en comunidad integrada por lo humano y lo natural-interelacionadas. (Carpio, 2014:96 - 97).

Para lograr el buen vivir son necesarios los valores culturales para que la convivencia y la complementariedad se realicen armónicamente en beneficio de los pueblos y comunidades. Recordemos que, la cultura puede transformar el contexto y fortalecer la comunidad a través de la autogestión con miras a satisfacer las necesidades y el logro de metas individuales y colectivas. Por consiguiente, el concepto de buen vivir debe incluir valores ético, normas, ideas y comportamientos que faciliten la armonía entre los seres humanos y la naturaleza.

\section{c. Ciudadanías Interculturales de Género}

Según el diccionario de educación para el desarrollo (Gema y López, s.f.) en su acepción más común, ciudadanía significa poseer la condición de ciudadano o ciudadana, entendiendo por tal la persona que es sujeto de derechos políticos en un estado y normalmente habitante de él. Lister (1997:41) refiere: "Ser un ciudadano o ciudadana en el sentido legal y sociológico implica poder disfrutar de los derechos de ciudanía necesarios para la agencia y participación social política.

Otro significado de la ciudadanía entendida como práctica activa y responsable es el de ciudadanía intercultural (Cortina, 2002), donde lo relevante es el respeto a las culturas que conduzcan a un diálogo desde el cual los ciudadanos pueden discernir qué valores y costumbres merecen la pena reforzar y cuáles obviar. Por tanto, consiste en la participación real y efectiva de las personas en la construcción y transformación de la sociedad. Se realiza a partir de la asunción de los derechos y deberes para incorporarlos a todos los ámbitos de la vida cotidiana. 


\section{d. Decrecimiento}

El decrecimiento es a la vez una crítica y una propuesta de alternativa a la sociedad del crecimiento. Se basa en la disminución regular y controlada de la producción, con la finalidad de establecer una nueva relación de equilibrio entre los seres humanos y la naturaleza (De María, Schneider, et al. 2018). Representa una dirección deseada, en la que las sociedades consumirán menos recursos y se organizarán y vivirán de modos distintos a los actuales donde el compartir, la convivencialidad y simplicidad son significados esenciales para definir el aspecto que tal sociedad tendría.

\section{e. Enfoque de derechos humanos}

El enfoque basado en los Derechos Humanos (Human Rights based approach, HRBA) está fundamentado en los valores, principios y normas universales propios de la dignidad de la persona humana (vida, libertad, igualdad, seguridad, participación política, bienestar social) y cualquier otro aspecto ligado al desarrollo integral de la persona y que se sustentan en los derechos humanos. Busca construir mecanismos jurídicos y políticos que transformen las instituciones, y consecuentemente la vida social y cotidiana de las personas con base en una nueva ética del desarrollo humano.

Considera a los pueblos indígenas, afrodescendientes, mestizos y comunidades étnicas de la Costa Caribe nicaragüense como agentes principales de su propio desarrollo mediante la participación efectiva de los hombres y mujeres en la transformación de su entorno para acceder a un mejor nivel de vida. Lo anterior se materializa a través de estrategias que promocionan el empoderamiento de los pueblos y comunidades mediante el acompañamiento comunitario para el fortalecimiento de la ciudadanía intercultural (URACCAN, 2017). Implica poner el foco de atención en los sectores históricamente excluidos o discriminados, requiere de un análisis de las normas de género, de las diferentes formas de discriminación y de los desequilibrios de poder, a fin de garantizar las estrategias de transformación pertinentes.

\section{Interculturalidad}

La interculturalidad envuelve la interacción entre culturas en los espacios definitorios de los órganos sociales y políticos. No sólo reconoce la diferencia, sino que hace énfasis en lo que las diferentes culturas tienen en común. Reconocer los vínculos que los unen, implica la promoción sistematica y gradual, desde el Estado y la Sociedad Civil, de espacios y procesos de interacción positiva que vayan abriendo y generando las relaciones de confianza, reconocimiento mutuo, comunicación efectiva, diálogo y debate, aprendizaje e intercambio, cooperación y convivencia, entre otros aspectos. (Valdillo Pinto, 2006). 
La interculturalidad, pensada y gestionada desde los movimientos sociales como un desplazamiento hacia afuera que requiere, en primera instancia, el fortalecimiento del núcleo cultural y social propio, antes de entrar en contacto con lo distinto. Apunta hacia la lucha por la simetría y la justicia.

Fornet-Betancour (1994) indica que, el diálogo intercultural no puede estar dominado o colonizado culturalmente por ninguna cultura especifica (...) propone renunciar al pensamiento único, basado en un solo modelo teórico y conceptual que sirve de paradigma interpretativo y abrirse a un dialogo basado en la relación igualitaria de los distintos logos que intervienen a partir de la asunción de la legitimidad de cada uno de ellos².

\section{Principios y valores que orientan el acompañamiento comunitario}

Es el conjunto de valores, creencias, normas, que orientan y regulan la vida de de la comunidad que se manifiestan y se hacen realidad en nuestra cultura, en nuestra forma de ser, pensar y conducirnos. Están presentes en cada uno de los procesos, programas, proyectos, actividades y acciones que realizamos de cara a dar un servicio de calidad a la sociedad. A continuación se señalan:

- Autonomía: Es la capacidad de autogobierno y autodeterminación, a nivel comunal, territorial, municipal, regional e internacional, que conlleve al buen vivir de los pueblos culturalmente diferenciados en unidad en la diversidad, así como a disponer de los medios para financiar sus funciones autónomas.

- Complementariedad: Es la comprensión y actuación de forma conjunta y colaborativa de hombres y mujeres que conlleva a la unidad y equilibrio entre ellos y su entorno para potenciar sus capacidades y alcanzar las metas como individuos, comunidad y pueblos.

- Comunitariedad: Es un proceso permanente de acompañamiento con calidad, de doble vía, en la construcción de planes, proyectos, estrategias, acorde a los modos de vida de las comunidades y pueblos para el buen vivir en correspondencia al modelo de universidad comunitaria intercultural.

- Conciencia ambiental: Es el sentido de valoración intrínseca y protección de la biodiversidad y ecosistemas naturales que se expresa con acciones constantes y sostenibles para garantizar la calidad de vida humana en equilibrio y armonía con la Madre Tierra.

- Corresponsabilidad: Es la suma de voluntades, de esfuerzos y recursos que tienen como meta el diseño y el cumplimiento de reglas que son de interés general fundamentada en una democracia participativa.

2 Citado en Rodríguez Triana, Tania (2019) Construcciones de paz con poblaciones indigenas en contextos interculturales. Guía para una cooperación sostenible al conflicto. Universidad del País Vasco, Instituto de Estudios sobre Desarrollo y Cooperación Internacional - HEGOA, España. Pag.16. 
- Consentimiento libre, previo e informado (CLPI): Es la aceptación manifestada por los pueblos indígenas, afrodescendientes, mestizos y otras comunidades étnicas en asambleas o cualquier otra forma de organización social, o por medio de sus representantes autorizados, sobre asuntos de interés de la colectividad, sometidos a su conocimiento, para cuya validez y legitimidad deben reunirse los requisitos y condiciones establecidos por instrumentos internacionales de derechos humanos y derechos colectivos.

- Diálogo de saberes y haceres: Es una práctica o proceso organizado de análisis que propicia la creación, recreación, diseminación e intercambio de conocimientos, sentires, sabidurías, saberes y haceres, en la búsqueda permanente del buen vivir personal, familiar y comunitario, desde la armonía entre el conocimiento propio y el occidental, con o sin la presencia de la Universidad, donde prevalece el valor de la palabra y la escucha respetuosa para alcanzar la comprensión común y la plenitud de la vida.

- Interculturalidad: Concebida como un proceso permanente de construcción, establecimiento y fortalecimiento de espacios de diálogo, comunicación e interacción horizontal de doble vía, entre personas, comunidades y pueblos de diferentes culturas. Su finalidad es la de la promoción y práctica de la equidad, el respeto, la comprensión, la aceptación mutua y la creación de sinergias para el establecimiento de sociedades inclusivas, no discriminatorias y libres de racismo. La interculturalidad comprende el desarrollo de procesos de toma de decisiones conjuntas en igualdad de condiciones.

- Solidaridad: Es la sensibilidad, empatía y colaboración recíproca de los miembros de grupos u organizaciones para apoyarse unos a otros y ayudarse mutuamente, surge de los intereses comunes y se basa en un sentimiento de pertenencia que favorece el desarrollo individual y colectivo.

\section{Sobre el acompañamiento comunitario intercultural}

\section{¿Que nos planteamos en un proceso de acompañamiento?}

El acompañamiento debe ser visto como un proceso formado por distintas etapas no lineales, pero si progresivas y continuas, la cual debe girar alrededor de las decisiones y pasos que van tomando en función de las capacidades que se desarrollan. Para ello, debemos definir qué tipo de acompañamiento estamos en la capacidad de brindar como Universidad que pueden estar referida a necesidades concretas o procesos más largos. Con la parte acompañada supone partir de las capacidades hacia el afianzamiento de sus potencialidades; con el acompañante se orienta la toma de decisiones, anima la realización de pequeños pasos, propone ideas y es el hilo conductor, un referente que posibilite un acompañamiento en el proceso de desarrollo. 


\section{CIENCIAS SOCIALES}

En este caminar, debemos de brindar especial atención a la utilización de un lenguaje inclusivo, que nos permita visibilizar la situación en la que se encuentran esos grupos específicos. Esta relación nos permitirá construir puentes de confianza a través del cual se gesta los vínculos que facilitarán el abordaje en profundidad de los procesos que desarrollamos con el protagonismo de las comunidades y pueblos. Proceso que debe de realizarse en todas las dimensiones ya que esta no puede centrarse en elementos parciales de la persona, sino que tiene que abarcar todas las dimensiones (física, cognitiva, psicológica, social y espiritual), se toma en cuenta las necesidades, el sentido y la participación de la persona y la comunidad.

Nuestro acompañamiento también tiene que propiciar que las personas se hagan preguntas sobre el sentido de su vida, sobre aquello que les motiva a vivir cada día y les da fuerza y aliento para superar las dificultades del camino. Descubrir esta motivación interior será el motor para activar todas las potencialidades a desarrollar en el proceso de acompañamiento. Es reconocer sus capacidades para comprometerse en la mejora de su situación y de su entorno y animarles a que las pongan en práctica.

\section{¿Qué cualidades necesitamos para realizar un buen acompañamiento?}

Es deseable que durante el proceso de acompañamiento las partes contemos con una adecuada combinación de las siguientes actitudes y capacidades:

\section{Actitudes:}

- Respeto a la autonomía de la persona y comunidad.

- Creer en la posibilidad de avance, de cambios.

- Escucha, responsabilidad, sentido común, coherencia. Sabe estar en el lugar que nos corresponde en cada momento.

- Flexibilidad, saber adaptarse a los cambios según las necesidades o situaciones que se va presentando.

- Con una mirada crítica y autocrítica.

- Sensibilidad y paciencia ya que estos procesos requieren tiempo.

- Apertura a nuevos aprendizajes, a compartir nuevas experiencias.

\section{Capacidades:}

- Saber trabajar en equipo.

- Empatía, saber ponerse en el lugar de la otra persona.

- Reconocer nuestros límites y aceptarlos.

- Saber escuchar, poder acoger lo que la persona expresa, necesita. 
- Saber comunicar, vincular, manejar las potencialidades.

- Saber reforzar, motivar, transmitir confianza.

- En los momentos que se necesite, saber poner límite.

- Saber consensuar, pactar, escoger cuales son las prioridades.

En el proceso de acompañamiento, necesitamos primero conocer nuestro caminar que nos ayudará a descifrar el caminar de la otra persona, sus necesidades, sentimientos, capacidades y posibilidades, obstáculos y tropiezos. Debe ser un acercamiento con respeto donde el diálogo de saberes y haceres nos permitirá conocer la realidad de la persona.

\section{Modelo de acompañamiento comunitario desde la mirada del post desarrollo}

\section{Sentido de comunidad}

Permite un arraigamiento en la consciencia colectiva de una seguridad de pertenencia, confianza entre los otros y una necesidad intrínseca de interdependencia, posibilita que las personas y grupos inicien y sostengan acciones colectivas orientadas a su bienestar, tanto a corto como a largo plazo. En la acción colectiva, la agencia comunitaria, el sentido de comunidad y la percepción colectiva de eficacia se refuerzan y potencian mutualmente (Saballos, 2017:86).

Los miembros de la comunidad reconocen la existencia de una conexión emocional, lazos compartidos como resultado del contacto positivo prolongado y de participar de experiencias y una historia común.

\section{Liderazgo comunitario}

El liderazgo es la fuerza motriz del desarrollo y el canal a través del cual fluyen las características para mejorar las capacidades de la comunidad para producir resultados mediante la utilización de los recursos que dispone (Banyai, 2009:59) ${ }^{3}$.

En liderazgo comunitario como actividad colectiva se construye y fortalece con la participación de cada uno de los miembros. Es un proceso de construcción del poder y la identidad comunitaria, puesto que promueve la democracia participativa mediante el poder del pueblo, en el que la mayoría de los ciudadanos se involucra en el tratamiento de problemas sociales críticos, liderando el cambio social lo que fortalece la ciudadanía, y que existe cuando una comunidad puede influir en las decisiones

3 Citado Rojas Andrade, Rodrigo (2013) El liderazgo comunitario y su importancia en la intervención Comunitaria, en sicología para América Latina (2013), 25, 57-76. 


\section{CIENCIAS SOCIALES}

tomadas por agentes externos del sector público y privado, y cuando puede acceder a recursos económicos y de otro tipo para lograr su propia agenda (Rojas, 2013:65).

\section{Apropiación comunitaria}

Es un estado de realización en el cual la comunidad, reflejando su voluntad en el liderazgo y organizaciones de base, ejercen su autonomía efectiva en su contexto de desarrollo, incluyendo los distintos procesos e iniciativas de cooperación externa que afectan sus vidas. Implica la comunidad asumiendo responsabilidad y control de los procesos de gestión relacionados a las distintas iniciativas de desarrollo, pudiendo variar su intensidad en las distintas fases involucradas (de Valk et al., 2005) ${ }^{4}$. Se logra a través de una organización comunitaria en donde los miembros de la comunidad se unen para analizar y buscar soluciones de manera conjunta con miras a lograr metas en común, fortalecer las capacidades de liderazgo dentro de la comunidad y aumento del capital social para lo cual se requiere de un acompañamiento sistemático con miras a asegurar una acción colectiva, proactiva, sostenida, participativa y democrática.

En el acompañamiento comunitario intercultural se debe tomar en cuenta la organización comunitaria y los fines que ésta persigue: apropiación comunitaria, fortalecimiento de habilidades y destrezas de las personas, es decir, debe tener presente el plan de vida de la comunidad que contiene sus sueños y aspiraciones. Implica una participación activa y propositiva de los miembros de la colectividad para así avanzar en la comprensión y transformación de su entorno social.

\section{Conciencia ambiental}

Es el entendimiento que tenemos como individuo y colectividad del impacto de los seres humanos en el entorno; cómo influyen las acciones de cada día en el medio ambiente y como afecta nuestro futuro. Para la preservación del medio ambiente se requiere de una actitud responsable en el uso de los recursos naturales que son factores fundamentales para poder preservar la naturaleza, la biodiversidad y por ende el futuro de la humanidad.

La sostenibilidad es fundamental, va desde la idealización hasta la implementación de las buenas prácticas que contribuyen a establecer una nueva relación de equilibrio entre los seres humanos y la naturaleza donde las ssociedades consumirán menos recursos, se organizarán y vivirán de modos distintos a los actuales donde el compartir, la confidencialidad y simplicidad son significados esenciales para definir el aspecto que tal sociedad tendría.

4 Citado en Saballos Velásquez, José Luis (2016) Hacia un Modelo Integral de Promoción de la Apropiación Comunitaria en las Regiones Autónomas de Nicaragua. Tesis Doctoral, Universidad del País Vasco, España. Pág. 91 


\section{Establecimiento de redes}

Los procesos de acompañamiento no pueden ser una acción aislada, sino un proceso colaborativo donde intervienen una serie de actores, siendo necesario:

- Conocernos mutualmente, para identificar aquello que nos une con el objetivo de sumar esfuerzos, complementarse (agenda de trabajo).

- Trabajar de manera articulada, abordando los problemas que van surgiendo y la valoración de las posibles acciones.

- La confianza, apoyo y refuerzo mutuo, así como el monitoreo y seguimiento a los procesos.

\section{Implementación del modelo}

Para la puesta en práctica de esta propuesta se deberá promover una serie de articulaciones internas y externas siguiendo los principios de participación democrática, diálogo horizontal, construcción colectiva y corresponsabilidad que la comunidad demanda. Asi como la capacidad de analizar, aportar, debatir y sobre todo decidir el futuro de la comunidad.

Un aspecto fundamental en la articulación interna es el sentido de comunidad, ya que a través de ella se promueve los lazos de solidaridad, permanencia, integración y cooperación entre los integrantes de la organización comunitaria que facilita la ejecución de acciones y mantenimiento del interés colectivo. Implica la interrelación entre las diferentes estructuras que forman parte de la organización comunal así como el análisis, discusión y ejecución de las diferentes acciones que realiza en el marco de cumplimiento de sus funciones, previa realización de asamblea comunitaria como máxima autoridad ancestral. En este sentido, el tejido organizacional nos indica los caminos a seguir para el logro de las metas y propósitos planteados.

La articulación externa se desarrolla a través del líder comunitario como representante legal de la comunidad apoyado por la Junta Directiva en diálogo con actores externos pr establecer acciones en cuestiones ambientales, políticas, humanas y económicas para el fortalecimiento del sistema autonómico y buen vivir de los pueblos.

En todos los niveles, debe desarrollarse una horizontalidad en la toma de decisiones para que el acompañamiento pertinente y de calidad en correspondencia con sus planes de vida. En este sentido, el tejido organizacional que se construye y las estrategias a implementar definidas nos indica los caminos a seguir para el logro de las metas y propósitos planteado en el modelo. 


\section{Sostenibilidad}

Para la sostenibilidad del modelo de acompañamiento comunitario intercultural desde elementos postdesarrollistas se debe incorporar acciones que conlleven a tal fin, a saber:

- Consolidación de la estructura comunal ancestral con las funciones, derechos, deberes y responsabilidades claramente definidas.

- Fortalecimiento de capacidades de líderes y lideresas en la gestión de proyectos, programas o procesos tomando en cuenta los principios y valores de este modelo donde el cuido de la Madre Tierra y su importancia para la pervivencia como pueblos.

- Establecimiento de redes con ONGs, universidades, instituciones del Estado y otras instancias con miras a la colaboración en cuestiones que contribuyan al fortalecimiento de las capacidades locales.

- Intercambio de experiencias con territorios o comunidades para conocer las buenas prácticas y los desafíos que enfrentan en la adopción de la propuesta de buen vivir desde elementos postdesarrollistas y dejar de lado aquellas que la afectan desde el punto de vista generacional y de género.

- Análisis del contexto socio-político para lograr mayor impacto de las actividades planteadas por los miembros de la comunidad. 

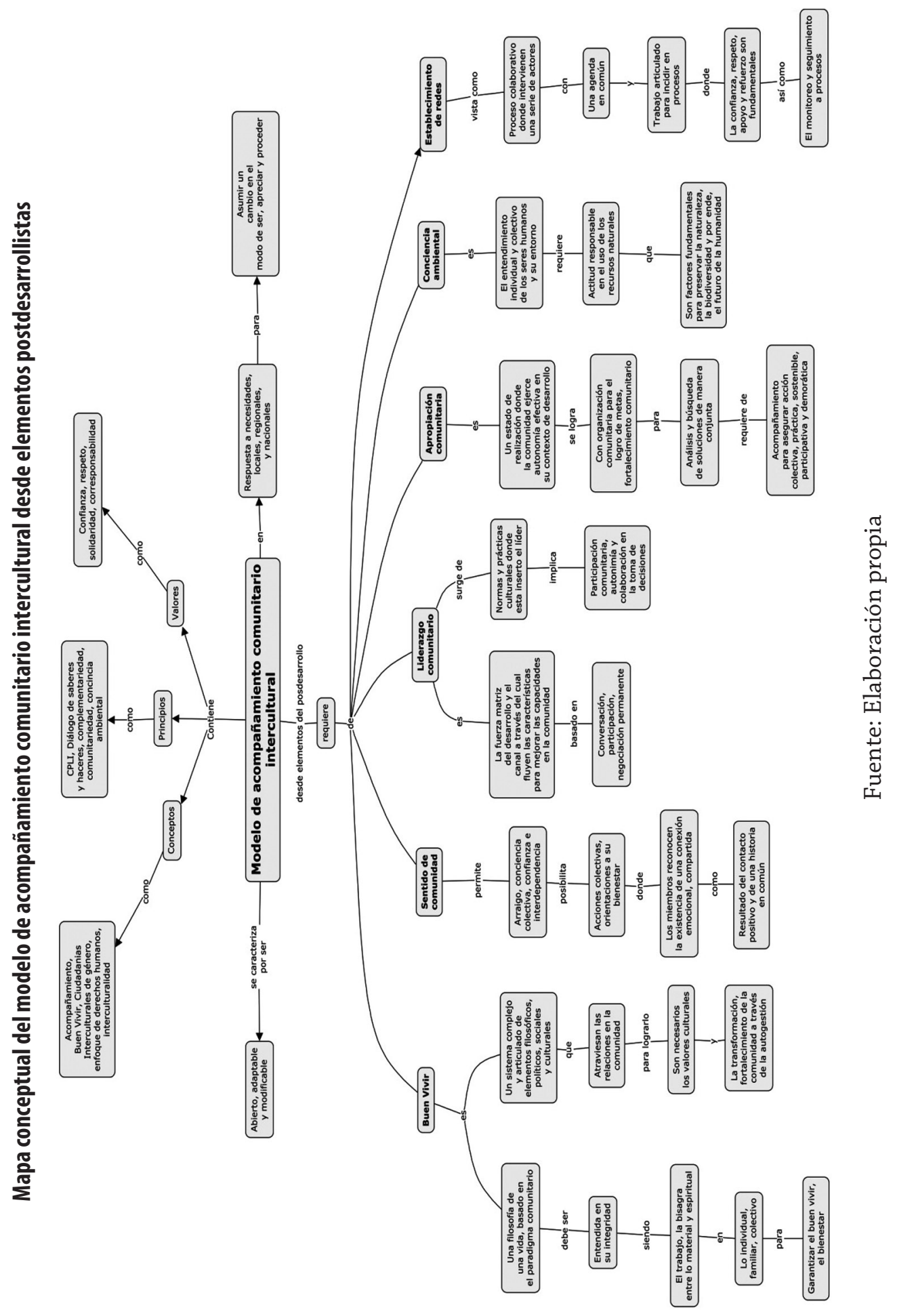


\section{Conclusiones}

Al hablar de un modelo de acompañamiento intercultural desde un enfoque posdesarrollista, debemos de identificar cuáles son los aspectos fundamentales a tomar en cuenta para acompañar a los Pueblos y Comunidades.

Para la materialización de estas aspiraciones, el proceso de acompañamiento debe ser colaboratorivo, participativo, concertado e inclusivo, sustentado en el dialogo de saberes y haceres, construcción colectiva y corresponsabilidad que la comunidad demanda. Acampamiento que debe responder a las necesidades y prioridadades identificadas en Asambleas Comunales o Territoriales, según estructura tradicional donde también se define periodo de acompañamiento, funciones, derechos y deberes con la finalidad que tengan claramente establecidas su rol dentro de dichas estructuras y el tiempo de acompañamiento.

Por tanto, las partes deben tener la capacidad de analizar, de aportar, debatir y sobre todo decidir el futuro de la comunidad de manera conjunta en pro del bienestar de la misma sin comprometer la capacidad de generaciones futuras de satisfacer las suyas y establecer una nueva relación de equilibrio entre los seres humanos y la Madre Tierra para lograr la vida en plenitud como filosofía de vida.

\section{Agradecimiento}

Esta publicación obtuvo el financiamiento de: El Fondo de Asistencia Internacional de los Estudiantes y Académicos Noruegos (SAIH).

\section{Lista de referencia}

Acosta, Alberto (2008). El Buen Vivir, una oportunidad por construir. Revista Ecuador Debate, n.75: 33-48.

Carpio Benalcazar, Patricio (2014). El buen vivir, entre la modernización capitalista y post desarrollo. Revista Papeles $N^{\circ} 128$ 2014/15, pp. 89-106. Centro de Investigación para la paz (CIP - Ecosocial), España.

Celorio Gema y López de Munain, Alicia (s.f.). Diccionario de Educación para el Desarrollo. Universidad del País Vasco, Instituto de Estudios sobre Desarrollo y Cooperacion Internacional - HEGOA, España.

De María, Federico; Schneider, Francois et al (2018). ¿Qué es el decrecimiento? De un lema activista a un movimiento social. Revista de Economía Critica, No 25, primer semestre 2018. 
Raya Diez, Esther y Hernández, Manuel (2014). Acompañar los procesos de inclusión social. Del análisis de la exclusión a la intervención social. Revista Trabajo Social 16: 143-156. Bogotá: Departamento de Trabajo Social, Facultad de Ciencias Humanas, Universidad Nacional de Colombia.

Rojas Andrade, Rodrigo (2013). El liderazgo comunitario y su importancia en la intervención Comunitaria, en sicología para América Latina (2013), 25, 57-76.

Rodríguez Triana, Tania (2019). Construcciones de paz con poblaciones indigenas en contextos interculturales. Guía para una cooperación sostenible al conflicto. Universidad del País Vasco, Instituto de Estudios sobre Desarrollo y Cooperación Internacional - HEGOA, España.

Saballos Velásquez, José Luis (2016). Hacia un Modelo Integral de Promoción de la Apropiación Comunitaria en las Regiones Autónomas de Nicaragua. Tesis Doctoral, Universidad del País Vasco, España.

URACCAN (2015). Plan Estratégico Institucional 2015-2019. Managua Nicaragua.

URACCAN (2017). Política de Acompañamiento Comunitario de URACCAN. Managua Nicaragua. 